\title{
Editorial
}

\section{Identifying Symptoms in Diagnostic Labyrinths: Adaptative Beacons from Biomarkers and Genetic Polymorphisms}

The latest International Meeting in the Series, "Strategies for Studying Brain Disorders", initiated in Madrid over fifteen years ago, maintained on a Biannual basis in Mojacar, and venued currently in Mazagon entitled, "Beyond Neuropsychiatric Diagnostics: Symptoms not Disorders", must attest to a singular propensity for establishment and continuity of frontline scientific endeavour, on the one hand, and a remarkable continuity of purpose, on the other, by Tomas Palomo, the Cerebro y Mente Fondacion, and the International Scientific Committee. Here, the different symptom profiles that partially characterise putatively shared and/or differentiating aspects of the disease process with highlighted, described and examined from clinical, non-clinical and basic experimental perspectives. The primary ambition and expected scope of the Meeting was to overcome the rigidly corseting carapace of existing diagnostic classifications of mental disorders to pave opportunities for overturning current prevailing notions.

An examination of common neurocircuits underlying common symptoms in neuropsychiatric disorders, particularly those arising from neurodevelopmental interference, was eminently appropriate to the initiation of the Meeting. One neurodevelopment approach to the understanding of schizophrenia was offered by the methylazoxymethanol acetate (MAM) model, consisting of the application of mitotoxin-induced hyperinnervation that produces a wide-range of functional, hyperactivity, maze learning and differential-reinforcement-oflow-rate operant schedules, and neurochemical, catecholamine hyperinnervation in the cortex and hippocampus, aberrations ${ }^{1}$. This technique was employed by Anthony Grace to study the hyperresponse of dopamine (DA) neurons underlying psychotic states in conjunction with hippocampus involvement through hyperactive ventral subiculum hippocampal neurones corresponding to the loss of parvalbumin-containing neurons $\left(\mathrm{Ca}^{++}\right.$-binding $)$. Circuitry involving the prefrontal-Nucleus Accumbens-Ventral Pallidum-Medial dorsal nucleus with inputs from the amygdala and hippocampus, incorporating 'spontaneously-active' and 'silent' DA neurons was outlined. Stimulation of the ventral subiculum of the hippocampus induced activation of 'silent' neurons with nucleus accumbens and ventral pallidal involvement, as well as determining the proportion of DA neurons firing. The presented results emphasised the direct links between hippocampal neuronal pathophysiology, alterations expressed in interneurons and the induced hyperdopaminergic MAM-status reminiscent of a postulated parallel status in schizophrenic patients. The intimate role of the dorsolateral prefrontal cortex (DLPFC) in the cognitive deficits, expressed for example in working memory impairments and identified as the neuropathological entity, was the point-of-departure for 
David Lewis and co-workers. The reduced mRNA expression of glutamic acid decarboxylase (GAD67), a synthesising enzyme for GABA, and the GABA membrane transporter (GAT1) are reduced in the subpopulation of GABA neurons that express calcium-binding protein parvalbumin. Changes in gene expression are linked to schizophrenia disease progression and appear not to be consequences of treatment but there are complementary changes, including reduced parvalbumin and GAT1 expression and up-regulation of postsynaptic GABA receptors. Working memory loading leads to gamma band power increased oscillations, an expression of the task and a feature disrupted in schizophrenics. Finally, the contributions of CB1-cannabinoid receptors was described. Francisco Artigas and co-workers maintained the cognitive theme of schizophrenic prefrontal cortex abnormality, examining the effects of PCP on prefrontal functioning to elucidate cellular and network elements contributing to this model of the disease. The contributions clozapine-mediated changes in serotonin neurotransmission that modulate prefrontal cortex-thalamic-ventral pallidal-hippocampal-amygdala-nucleus accumbens interacting with NMDA receptors provided the platform for the electrophysiological counterparts of working memory, executive function, etc. PCP induced a marked disruption of neuronal activity in the rat prefrontal cortex, reducing too cortical synchrony in the delta frequency range $(0.3-0.4 \mathrm{~Hz})$, DOI was found to produce the same effects and antipsychotic agents blocked both the effects of PCP and DOI. The neurodevelopmental aspect of schizophrenia was maintained by Jose Borell describing the loss of sensory gating ability, as indexed by PPI, following administration of the bacterial endotoxin LPS to pregnant rat dams throughout gestation and assessment of the offspring with regard to PPI, dopaminergic function, brain protein expression and cytokine serum levels from weaning to late adulthood. Age-specific changes were obtained in accumbal DA levels and protein expression in the frontal cortex, as well as age-dependent and cytokinedependent alterations in serum cytokine levels. These results presented important insights on the immune system contributions to the etiology of schizophrenia. The hypofunctioning of NMDA receptors holds consequences for eventual cognitive symptom profiles, including executive function, as outlined by Bita Moghaddam and co-workers, who described the effects of use-dependent inhibition of NMDA receptors on the spontaneous activity of putative GABA interneurons and pyramidal neurons in the prefrontal cortex of awake rats. Inhibition of NMDA receptors decreased activity of the putative GABA interneurons but increased that of the majority of pyramidal neurons. Their findings offer a clinically relevant mechanism whereby the resulting hyperactivity of pyramidal neurons and excess glutamate "spill-over" may be applied to the search for novel antipsychotic agents. Michael Sutton demonstrated that mode of neurotransmission may exert much influence on ongoing protein synthesis in dendrites, posing the question: how does one maintain the stability of activation in neural circuits? He described recent studies implicating local dendritic protein synthesis in many types of synaptic plasticity with regard to how neurotransmission affects ongoing protein synthesis in dendrites. Thus, the tetrodotoxin-blockade of intrinsic action potential mediated network activity in cultured hippocampal neurons inhibited the dendritic synthesis of a fluorescence translation reporter, with ongoing spontaneous neurotransmitter release bidirectionally regulating dendritic protein synthesis.

Current notions of symptom categories in psychosis and schizophrenia would appear to lean towards the distinctions arising from Positive - Negative - Cognitive - Affective pro- 
files, thereby rendering constituted array of meeting presentations eminently suitable in particularly relation to genetic predispositions ${ }^{2}$. Mark Geyer discussed aspects of the neurobiological substrates, constructs, mechanisms, relevance of effects, parallel effects in transitional models, etc, underlying the development of animal models to mimic diagnostic syndromes in the search for models of specific symptoms. The applications of prepulse inhibition for the examination of sensory gating was exploited as a case in point whereby strain differences in prepulse inhibition, with or without clozapine/haloperidol administration, was exemplified. Illustrations of more or less valid cross-species models, specific animal paradigms that parallel pre-cognitive / cognitive expressions and development of novel human models of neuropsychiatric symptoms were provided. Celso Arango presented a discourse on the shared genetic susceptibility and partial overlap between bipolar disorder and schizophrenia, the distinctions in the course of disease, functional impairments and the response to treatment. In identifying patterns of cognitive deficit, e.g. involving selective attention, verbal memory and executive function, associated with specific psychiatric disorders whereby the disorders present a qualitatively similarly profile of deficit, the backdrop to neuropsychological functioning among patient subgroups concerning Early Onset Psychosis (EOP) was outlined ${ }^{3}$. The results, from a large first episode EOP population, characterised the attentional, working memory, verbal memory and executive function deficits but nevertheless a lack of specificity in the cognitive impairment. The implications of longitudinal studies that take into account eventual 'improvement effects' are awaited. Anissa Abi-Dargham and co-workers, with a positive-negative-affective-cognitive reflecting positive symptom profiling juxtapositioned with pre- and postsynaptic excess striatal DA, described implications of significant increases of the DA D1 receptor in the dorsolateral prefrontal cortex, e.g. in DA-depleted rats there was increased D1 over all regions including the striatum, prefrontal cortex and hippocampus. They observed that under schizophrenia-like conditions, e.g. ketamine-users/administration and COMT gene high activity (Val/Val carriers), DA transmission was increased in the associative striatum at the head of the caudate the area of projection to the dorsolateral prefrontal cortex. Treatment response was related to the degree of DA D2 occupancy in mesolimbic and associative striatal but not extrastriatal areas. Douglas Blackwood and co-workers described family linkage studies that have identified a number chromosomal locations likely to harbour genes contributing to symptoms of disorder but few linkage "hot spots" wherein meta-analyses have been performed. Several complicating issues were touched upon, including heterogeneity of the disease process in schizophrenia, learning disabilities and psychotic episodes among patients, family history accompanied by intellectual impairments that together with structural abnormalities gave considerations of lower-higher rates of chromosomal abnormalities. Interestingly, rather strikingly similar patterns of neuropsychiatric symptom profiles were observed in patients with different genetic abnormalities. Analyses of the phenotypes supported the notion that similar symptom patterns may develop multi-disrupted brain pathways via multiple mechanisms. Sigfried Tunier and Willem Verhoeven discussed the genetic underpinnings of schizophrenia from the perspective of a broad clinical phenotype regarding developmental issues, symptoms and co-morbidity. From the case of 22q11 deletion syndrome, psychotic symptoms emerge from a context of brain anomalies, an interaction of somatic abnormalities and specific neurocognitive deficits whereas in the PraderWilli syndrome, a hypothalamic disorder is expressed by psychotic symptoms that may resemble Schizophrenia. Jose Luis Vazquez-Barquero and co-workers examined the effec- 
tiveness and tolerability of antipsychotic treatment of drug naïve first episode psychotic patients from a perspective of adherence and compliance, care-giving variables, long-term outcome and eventual burdens on relatives. The efficacy of generations of anti-psychotic agents was derived from the Cantabrian First Episode Psychosis Clinical Program (PAFIP) from 172 drug naïve patients assigned to haloperidol, olanzapine and risperidone treatment regimes. It was found that under routine clinical conditions relatively low doses of all three antipsychotic agents were equally effective in treating first episode psychotic patients acutely, albeit the side-effect profiles of each were markedly different. Robin Murray and Paul Morrison with co-worker discussed the likelihood of schizophrenia-like psychoses in the general population from observations of individuals using illicit drugs. Recent onset psychotic patients were twice as likely to be heavy users of cannabis as controls, and observations from the Swedish Army Study, Dunedin Study and several cohort studies all point in the same direction: Cannabis increases the risk for psychosis. Nevertheless, only some users develop psychosis: statistically, $4.7 \%$ of those debuting substance use by 18 years and $10.3 \%$ of those debuting by 15 years-of-age. Gene-environment interaction studies implicate the COMT and/or DRD4 gene to predispose. Finally, they outlined a study pertaining to incidence of PANSS in drug users and controls.

The contributions of cognitive symptoms, whether modulated by or modulating affective states, to the pathophysiology of brain disorders was showed in both clinical, non-clinical and laboratory settings. Antonio Lobo and co-workers set out to study the power of psychopathological, non-cognitive symptoms to predict incident cases of mild cognitive impairment (MCI) and Alzheimer's Type dementia in a representative community sample of patients aged 55+ years, interviewed in a two-wave screening, and mainly applying the ZARADEMP Interview; two years later ZARADEMP II was applied. Their results showed that several non-cognitive symptoms, at baseline assessment, were predictive of MCI, e.g. irritability, neurovegetative symptoms, sleep disturbances, loneliness and subjective slowing, as well as Alzheimer's Type dementia, e.g. sleep disturbances, observed slowing and morbid thoughts. Thus, specific psychopathological and non-cognitive symptoms were predictive of MCI and Alzheimer's Type dementia in an elderly population. Jeremy Seamans and coworkers presented evidence of prefrontal cortex deficits underlying working memory problems in schizophrenia, posing the question of what is the loss of cortical efficiency? Their findings that a dysfunctional DA system contributes to both positive and cognitive symptoms were supported by the observation that amphetamine can both initiate/exacerbate psychotic symptoms and affect the efficiency of PFC circuits that process working memory. Amphetamine-induced alterations in the activity of multiple, simultaneously recorded PFC neurons in rats performing a working memory task. Schizophrenic patients tend to activate a much larger area of cortex implying greater cortical 'noise' and loss of signal-noise ratio thereby begging the question regarding how DA modulate $\mathrm{S}: \mathrm{N}$ in working memory tasks? It would appear that DA D1 receptor regimes give way to DA D2 receptor regimes thereby affecting efficiency and allowing stereotypy that interferes with the performance of working memory: as D2 > D1 regime develops performance decreases and working memory efficacy decreases. Jose Maria Delgado-Garcia described trace conditioning in the cerebral cortex, long-term potentiation (LTP) and long-term depression (LTD) that he has developed for studying learning and memory in wild type and transgenic (APP, PS1, TrkC, D1-D2, Pol $\psi$, etc) mice 
expressing cognitive deficits. He has evolved techniques for measuring field excitatory postsynaptic potentials (fEPSPs) evoked at selected hippocampal sites by electrical stimulation of monosynaptic afferent inputs. Taken together, the findings display a plethora of cognitive symptom patterns in genetically compromised mice presenting neuropsychiatric disorders involving cerebral cortex integrity. Lia Bevilaqua recounted the progress regarding the description and understanding of conditioning, testing and molecular processes underlying memory formation and expression in laboratory animals. She discussed several issues relating to the forgetting of information as well as biochemical processes specifically involved in memory persistence. Following this, Ivan Izquierdo and co-workers recalled progress and findings reported over the past decade demonstrating that the memory consolidation of onetrial inhibitory avoidance conditioning relies on a sequence of events in the CA1 region of the hippocampus almost identical to LTP-induction in that area. He described differences between long-term memory and short-term memory with regard to mechanisms pertaining to encoding, consolidation and expression. The notion of 'endogenous state dependency' incorporating the repetitions of neurohumoral conditions of training that are necessary for optimal behavioural performance - was discussed in the context of memory persistence - the relationships of remembering over time of training and test after training, and involved the role of BDNF infusions.

Movement disorders and/or motor expressions of neuropsychiatric symptoms may be associated generally with neurological disease states but nevertheless the accounts rendered below serve to illustrate the utility of maintaining neurologic perspectives on psychiatry, and vice versa. Manfred Gerlach and A. Warnke discussed the associations of the movement disorders with a broad spectrum of disorders defined by inability to initiate movement, hypokinetic disorders, at one end of the scale, and conditions encompassing an exaggeration of motors behaviours, hyperkinetic disorders, at the other end of the scale; the former case was exemplified by Parkinson's disease and similar neurodegenerative conditions and the latter by a constellation of disorders such as Huntington's Chorea and Hemiballism but also other neuropsychiatric conditions like Tourette's syndrome, Lesch Nyhan's disease, Attention Deficit Hyperactivity Disorder and Schizophrenia, often DA-glutamate interactions providing central features. One fundamental issue highlighted in this account pertained to utility of distinctions between psychiatry and neurology but rather the further development of neurobiological approaches. Richard Kostrzewa and co-workers maintained this broad neurobiological theme in exploring DA-receptor sensitivity in relation to stereotypic motor behaviours, in particular vacuous chewing movements (VCMs) and locomotion. They presented a series of results illustrating the influence lesioning of presynaptic terminals (e.g. applying postnatal 6-hydroxydopamine) or prolonged receptor antagonist action combined with acute administrations of DA D1 or DA D2 to elicit the behavioural responses. They suggested that DA receptor sensitivity and stereotypy (RSS) may reflect receptor subsensitivity and/or modulation by receptor co-sensitization of another phenotype. Moussa Youdim introduced and developed the contention, "dirty drugs for dirty diseases", currently an appealing concept in drug development, through descriptions of neurotoxic events, e.g. oxidative stress, excitotoxicity, inflammatory processes, brain iron dysregulation, etc, in the neurodegenerative disorders and the new therapeutic strategies under development. Here, candidate drugs have been designed to act on multiple neural and biochemical targets, combining cholinesterase inhibi- 
tion, monoamine oxidase inhibition, iron chelation, inhibitors of glutamate release and antiapoptotic neurorescue activity. These diverse activities were tested over a variety over animal and in vitro models as well as in transgenic mice.

Neuropathological levels of impulsiveness are linked to every instance of neuropsychiatric co-morbidity ${ }^{4}$, with 'inability to plan', 'lack of self-control' and 'novelty/sensation seeking' the most marked factors identifying the condition. Giovanni Laviola and W. Driani described laboratory markers for assessing impulsivity in adolescent rats (30 to 45 days of age) whereby in these rats impulsivity was measured using novelty-seeking behaviour and the elevated plus maze to indicate that adolescents exhibited greater impulsivity than adults. On the other hand, adolescents showed less hyporesponsivity to drug effects, e.g. amphetamine, than adults, as was observed too with place preference conditioning. In comparison with adults, adolescents showed "extremes-oriented" behaviour described by biomarkers indicating low metabolism/serotonin (5-HIAA/5-HT) ratio in the medial frontal cortex (MFC) and noradrenaline levels in the MFC and cingulated cortex. They suggest that a developmental rearrangement of forebrain systems may explain some of the observed behavioural anomalies. Janet Hoenicka outlined consequences of the Taq1A single nucleotide polymorphism, the most studied genetic variation implicated psychopathy, alcoholism and substance abuse disorders, abnormal P300 amplitude, affective disorders, etc. Nevertheless, DRD2 (the DA D2 receptor gene) Taq1A is not in fact located within DRD2 but within a protein coding region exon 8 of the novel adjacent gene, ANKK1 (Ankyrin repeat and kinase domain containing 1 protein), where it causes the missense substitution E713K. They have observed that the ANKK1 kinase is expressed in the cerebrum, cerebellum and spinal cord and may offer an important step towards understanding functional differences related to Taq1A SNP, underlying vulnerability. Cesar Avila and co-workers applied Reinforcement Sensitivity Theory to develop notions, arising from the concept of approach-avoidance, in appetitive contexts, pertaining to 'fight-flight', 'behavioural activation' and 'behavioural inhibition' and the particular role of serotonin. Direct modulation of brain activation during appetitive stimulus processing is expected from the brain activation system whereas inverse modulation during aversive stimulus processing is expected as a reflection of the system's antagonism. They concluded that sensitivity to reward may be construed to be one expression of impulsiveness related to both enhanced appetitive learning and poorer aversive learning. Rick Beninger discussed current notions regarding the acquisition and expression of incentive learning in relation to the role of DA, i.e. DA in acquisition and DA-antagonism in expression. A consensus of evidence highlights the position that DA acting via D1-like and D2 receptors is critical. Using a conditioned activity procedure based on cocaine it was found too that DA D2 and DA D3 receptors function differently, presenting a double dissociation with D2 receptors necessary for establishment, but not expression (initial), of conditioned activity; conversely, D3 receptors were necessary for expression but not establishment. Guillermo Ponce described characteristics involving C957T, a functional polymorphism of the DRD2 gene associated with phenotypic features previously linked to the A1 allele of the Taq1A polymorphism, and ANKK1 (see above). It was found that the CC genotype of the C957T SNP of the DRD2 gene, but not the A1+ genotype of the Taq1A SNP of the ANKK1 gene, was more prevalent in the whole sample of 176 male alcoholic patients in comparison with 150 healthy controls. Both an overrepresentation of the $\mathrm{CC}$ and $\mathrm{A} 1+$ genotypes was obtained in a group- 
ing of alcoholic patients according to presence of Dissocial Personality Disorder and a link to high scoring on the Psychopathy Checklist-R. Peter Kalivas treated the neuropsychiatric aspects of addiction from the perspective of two characterizations:

a) an inability to regulate cognitively the drive to obtain drug or habitual drug-seeking behaviour, and b) a reduced drive to obtain natural rewards together with increased drug-taking behaviour. The notions of regulated relapse and compulsive relapse were described from a viewpoint of 'key to treatment' and 'extinction responding after abstinence'. Loss/inability of prefrontal cortex capacity to guide behaviour seems a consequence of neuroadaptive changes in the prefrontal cortical output (glutamatergic, inactivated by GABA agonists) to the nucleus accumbens as a result of repeated drug use.

Anhedonia, characterised by lack of interest in life, the joy of living, and an incapacity to experience enjoyment, appears to comprise both affective, cognitive and particularly motivational components. Michel Le Moal addressed issues arising from the applications of animal models in behavioural pathology and experimental psychopathology, with particular regard to influences of the former. Most animal models appear not to fit the clinical features, and above all the clinical realities. Pathological traits or phenotypes characterise the co-existence of different modular components, each a symptomatic expression of dysfunction within a given 'anatomo-functional' system. Biological defects co-exist with pathobehavioural trait characteristics as signatures of different neurobiological systems. Ian Reid and coworkers indicated the commonness of early trauma, $6-62 \%$ of women and $3-31 \%$ of men, underlying a multitude of psychiatric health problems, including depression, PTSD, eating disorders, self-mutilatory behaviour, suicidality, etc. Thus, both prenatal and early postnatal stressors may induce long-lasting vulnerability, sometimes latent, resulting in adverse experience throughout adulthood. They report significant trauma histories across diagnostic groups (including schizophrenia, depressive disorders, bipolar disorder, anxiety disorder, substance misuse and borderline personality disorder). Alexander Mathe and co-workers discussed neuropathological consequences (e.g. clinical depression), that persist into adult life, of early life adverse events from a perspective of genetic and environment factors. Several parameters, including age, duration, type of event, event perception, defence mechanisms, caretakers, environment, variable measurements, etc, were implicated. The findings from an animal model paradigm, maternal deprivation during postnatal days 2-15 (15 min - 6 hour placement in a temperature-controlled incubator) showed altered behavioural responses to stress, HPA axis hyperactivity, dysregulated monoaminergic systems, as well as affecting neuropeptide $\mathrm{Y}$ and calcitonin gene-related protein. They compared these rats, Sprague-Dawley with rat lines showing affective problems thereby presenting the notion that genetic loading and developmental stress-trauma ensure susceptibility to clinical depression. Mary Phillips described functional abnormalities in neural systems underlying affect processing and vulnerability to anhedonia with regard to the concept of endophenotypes in the genotype-visible entity-bipolar disorder interaction. A neural model for emotion incorporating visual cortexthalamic(ventral striatum)-amygdala - orbitalfrontal cortex (the hinge) - hippocampusparahippocampus-dorsomedial prefrontal cortex-dorsolateral prefrontal cortex-ventrolateral prefrontal cortex circuitry was discussed in the light of specific abnormalities of neural systems and mood regulation. Roy Wise recalled that notions and reasoning, i.e. the role of DA in reinforcement and incentive motivation, underlying the Anhedonia hypothesis, referring 
not to the influence of DA in affecting 'ability to respond' but rather to 'maintain' rewardseeking behaviour. The hypothesis focussed attention on the apparent paradox that neuroleptic compounds attenuated reward-associated positive reinforcement in laboratory animals. The dominant role of DA in reinforcement, underlying the anhedonia hypothesis, is essential to response habits, conditioned preferences and synaptic plasticity in cellular models of learning and memory, and not least to neuroadaptative notions of addiction.

\section{References}

1. Archer T, Hiltunen AJ, Järbe TUC, Reza Kamkar M, Luthman J, Sundström E, et al. Hyperactivity and instrumental learning deficits in methylazoxymethanol-treated rat offspring. Neurotoxicol Teratol 1988; 10: 341-347.

2. Palomo T, Kostrzewa R, Beninger RJ, Archer T. Genetic variation and shared biological susceptibility underlying comorbidity in neuropsychiatry. Neurotoxicity Res 2007; 12: 29-42.

3. McClellan J, Prezbindowski A, Breiger D, McCurry C. Neuropsychological functioning in early onset psychotic disorders. Schizophr Res 2004; 68: 21-26.

4. Palomo T, Kostrzewa R, Beninger RJ, Archer T. Treatment consideration and manifest complexity in comorbid neuropsychiatric disorders. Neurotoxicity Res 2007; 12: 43-60.

Trevor Archer

Department of Psychology, Göteborgs University

SWEDEN 